\title{
Noise Figure of Silicon Raman Amplifiers
}

\author{
Dimitrios Dimitropoulos, Daniel R. Solli, Ricardo Claps, Ozdal Boyraz, Member, IEEE, and \\ Bahram Jalali, Fellow, OSA
}

\begin{abstract}
The noise figure of silicon Raman amplifiers in the presence of nonlinear losses is calculated. The impact of twophoton absorption (TPA) and free-carrier scattering on the noise figure is quantified using the quantum formulation of the Langevin approach. It is found that TPA-induced free-carrier loss degrades the noise figure by an amount that depends on the carrier lifetime. For example, in a 1-cm-long waveguide pumped at $200 \mathrm{MW} / \mathrm{cm}^{2}$, the noise figure is $5.2 \mathrm{~dB}$ for a lifetime of $\tau=1.6 \mathrm{~ns}$ and is reduced to $3.7 \mathrm{~dB}$ for $\tau=0.1 \mathrm{~ns}$. The reduction in the noise figure along with a concomitant increase in Raman gain from 2 to $8 \mathrm{~dB}$ suggests that lifetimes on the order of $0.1 \mathrm{~ns}$ or less are needed to create a useful silicon Raman amplifier that operates in the continuous-wave mode. It is also shown that in devices that use a p-n junction for carrier sweep-out, the screening of the junction field by generated free carriers results in a sharp increase in the noise figure at high-pump intensities. These results apply to operation in the near-infrared communication wavelengths. For mid-infrared wavelengths above the two photon absorption band-edge $(2.3 \mathrm{~nm})$, the absence of TPA and pump-induced free-carrier absorption ensures that the amplifier has a low-noise figure.
\end{abstract}

Index Terms-Amplifier noise, optical noise, silicon, silicon on insulator technology.

\section{INTRODUCTION}

$\mathbf{T}$ HE recent observations of high Raman gain [1]-[3] in bulk silicon along with demonstrations of Raman lasers [4], [5] have opened up new possibilities for low-cost photonic components that, in some cases, may be amenable to integration with CMOS electronics. For operation in the technologically important $1300-1550 \mathrm{~nm}$ wavelength range, the main challenge is the nonlinear optical loss that competes with the Raman gain. This loss is caused by absorption from free carriers that are created in the medium because of two-photon absorption (TPA) induced by the high-intensity pump beam (Fig. 1). The free-carrier absorption loss is proportional to the lifetime of the carriers in the amplifying medium. In this paper, we show, for the first time, how the nonlinear losses affect the signal-to-noise ratio of a Raman amplifier. We use the model to determine the minimum noise figure of the silicon Raman amplifier as a function of the carrier lifetime, waveguide losses, and pump intensity.

Manuscript received March 13, 2007; revised August 8, 2007. This work was supported by the MTO office of the Defense Advanced Research Project Agency (DARPA).

D. Dimitropoulos, D. R. Solli, and B. Jalali are with the Department of Electrical Engineering at the University of California, Los Angeles, CA 90095 USA (e-mail: ddimitr@ee.ucla.edu; solli@ucla.edu; jalali@ucla.edu).

R. Claps was with the University of California, Los Angeles, CA 90095 USA. $\mathrm{He}$ is now with Neptec Optical Solutions, Fremont, CA 94539 USA (e-mail: ricardoc@nepopt.com).

O. Boyraz was with the University of California, Los Angeles, CA 90095 USA. He is now with the University of California, Irvine, CA 92697 USA (e-mail: oboyraz@uci.edu).

Digital Object Identifier 10.1109/JLT.2007.915211

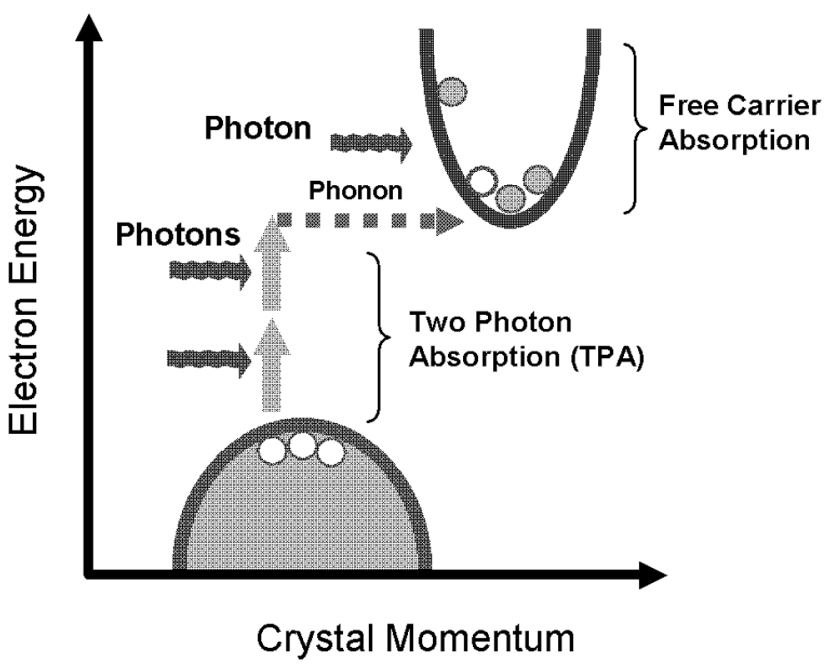

Fig. 1. Illustration of two-photon absorption and the resulting free carrier absorption when silicon is illuminated with a high-intensity pump beam.

\section{Derivation OF THE NOISE FIGURE}

\section{A. Propagation Equation for the Stokes Signal}

In a single-mode silicon waveguide Raman amplifier, the Stokes wave evolves according to the equation

$$
\frac{d}{d x} \hat{a}_{S}=\left(\frac{g(x)}{2}\right) \hat{a}_{S}-\left(\frac{\gamma^{\prime}(x)}{2}\right) \hat{a}_{S}
$$

where $\hat{a}_{S}$ is the photon annihilation operator for the Stokes field and $x$ is the position along the length of the waveguide. The operator is normalized according to the commutation relation $\left[\hat{a}_{S}, \hat{a}_{S}^{+}\right]=1$, where $\hat{a}_{S}^{+}$is the creation operator for the Stokes field. The gain parameter is given by $g(x)=g_{R} I_{P}(x)$, where $I_{P}(x)$ is the pump intensity and $g_{R}$ is the Raman gain coefficient. The incident pump wave amplitude is treated as a classical field (c-number) and its propagation is described later (5). The Stokes wave experiences losses determined by the coefficient $\gamma^{\prime}(x)=\gamma+2 \beta I_{P}(x)+\beta^{\prime} I_{P}^{2}(x)$. The first term characterizes the linear propagation loss, the second term includes loss from two-photon absorption (TPA), and the third term incorporates optical absorption from free-carriers generated from the TPA process. Although the linear loss is independent of the nonlinear terms, the coefficients of the two nonlinear loss terms above are dependent on each other. This connection can be demonstrated by examining the characteristics of the free-carriers created by TPA. The number density of TPA-generated electron-hole (e-h) pairs equals $R \times \tau$, where $R=\beta I_{P}^{2} /\left(2 E_{p}\right)$ is the number of pairs generated per unit time per unit volume, $E_{p}$ is the photon energy, and $\tau$ is the "effective" lifetime of the pairs. Given the optical absorption cross section $\sigma$ of an electron-hole pair, we 


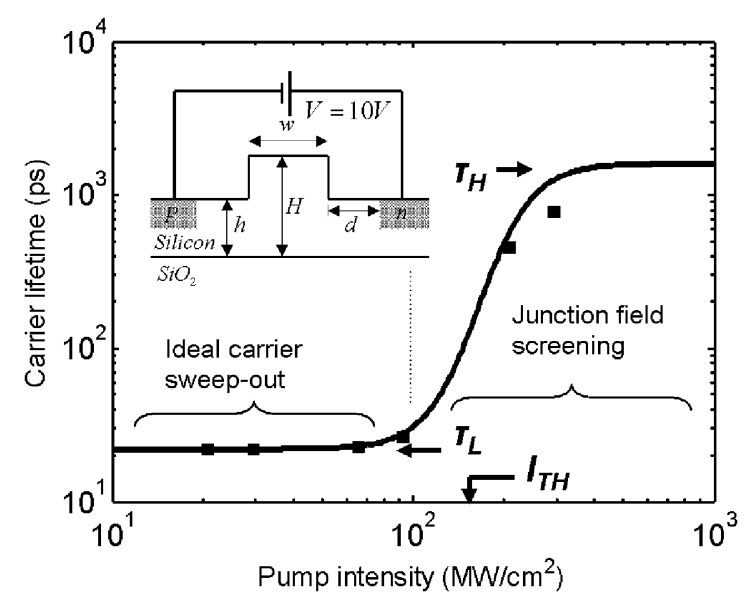

Fig. 2. Fit of the carrier lifetime with (2) to the simulation results (squares).

find $\beta^{\prime}=\beta \sigma \tau /\left(2 E_{p}\right)$. These nonlinear absorption terms are present in silicon Raman amplifiers and impact their noise performance characteristics.

In a Raman amplifier, the mechanism by which the TPA generated carriers exit the waveguide core is significant since it determines the effective carrier lifetime [6]. For continuous-wave (CW) devices, two mechanisms must be considered. First, carriers are removed from the waveguide by diffusion and by recombination. The latter occurs in the waveguide core, at the $\mathrm{Si}-\mathrm{SiO}_{2}$ interface, and at the silicon surface. In this case, the lifetime is largely independent of the carrier density and, therefore, from the pump intensity that produces the carriers [this fact is no longer true when the carrier density reaches the onset of Auger recombination $\left.\left(\sim 10^{18} \mathrm{~cm}^{-3}\right)\right]$. The second mechanism for carrier removal arises if an electric field is applied to sweep away the carriers. In particular, the carriers will drift in the presence of the electric field of a reverse-biased $\mathrm{p}-\mathrm{n}$ junction sandwiching the waveguide [5]. In this case, lifetimes on the order of $10 \mathrm{ps}$ or less can be achieved (for $1 \mu \mathrm{m}$ wide waveguide) but the lifetime now depends on the carrier density. At high-pump intensities, the increase in the carrier density gives rise to a screening of the applied electric field by carriers so that the lifetime is a function of the incident intensity $\tau=\tau\left(I_{P}\right)[6]$, [7]. The carrier lifetime is constant for low enough intensities, but when the optical intensity exceeds a given value, a sharp "step-like" increase occurs after which the lifetime is again independent of intensity. At this threshold, the carrier density becomes large enough that the carriers no longer drift under the action of the applied field and diffusion/recombination becomes the dominant transport mechanism [7]. In this paper, the function $\tau=\tau(I)$ is determined through numerical simulation of carrier transport since an analytic expression cannot be obtained. The function

$$
\tau(I)=\tau_{H} \times \exp \left(\frac{\left(\frac{I}{I_{\mathrm{TH}}}\right)^{n}-\xi}{\left(\frac{I}{I_{\mathrm{TH}}}\right)^{n}+1}-1\right)
$$

is an analytical fit to the numerical results. Here, $\xi=$ $\ln \left(\tau_{H} / \tau_{L}\right)-1$ and $\tau_{L}, \tau_{H}$ are the low- and high-intensity values of the lifetime. Fig. 2 shows the numerical results and the above analytical model. The exponent $n$ and the threshold intensity $I_{\mathrm{TH}}$ are the model fitting parameters.

\section{B. Langevin Noise Sources for the Silicon Raman Amplifier}

We now turn our attention to the noise of the silicon Raman amplifier. At thermal equilibrium the fluctuations of the field amplitude must maintain their equilibrium value along the waveguide length. In a lossy waveguide, this is accomplished by introducing the so called Langevin noise sources into the equation for propagation of the field amplitude [8].

The commutator of the field operators determines the noise limit imposed by the uncertainty principle. More specifically, $\left\langle\Delta \hat{a}_{I}^{2}\right\rangle\left\langle\Delta \hat{a}_{Q}^{2}\right\rangle \geq(1 / 4)\left\langle\left[\hat{a}_{I}, \hat{a}_{Q}\right]\right\rangle^{2}$, where $\hat{a}_{I}=(1 / 2)\left(\hat{a}_{S}+\right.$ $\left.\hat{a}_{S}^{+}\right)$and $\hat{a}_{Q}=(1 / 2 i)\left(\hat{a}_{S}-\hat{a}_{S}^{+}\right)$represent the in-phase and quadrature components of the electromagnetic field. Langevin noise sources are required to maintain the commutator at a constant value, i.e., $d\left[\hat{a}_{I}, \hat{a}_{Q}\right] / d x=0$, as required by the uncertainty principle. A more rigorous justification of commutator conservation is provided in [8].

For the loss and gain terms in the propagation equations, the required noise sources are given by the operators $\sqrt{\gamma^{\prime}(x)} \hat{N}_{L}$ and $\sqrt{g(x)} \hat{N}_{G}^{+}$, respectively, where

$$
\left[\hat{N}_{L}(x), \hat{N}_{L}^{+}\left(x^{\prime}\right)\right]=\left[\hat{N}_{G}(x), \hat{N}_{G}^{+}\left(x^{\prime}\right)\right]=\delta\left(x-x^{\prime}\right) .
$$

The derivation of (3) is provided in the Appendix. These noise operators act on "noise reservoir states." At every point in the waveguide, two noise reservoir states are defined: one for the gain and one for the loss. Each continuum of states is labeled by the position $x$ in the waveguide; the noise reservoir state, say for the gain, is denoted by |\rangle$_{G}=\prod_{x}|\rangle_{G, x}$, where |\rangle$_{G, x}$ is the noise reservoir state at position $x$. A similar expression applies for the loss reservoir.

At this point, we would like to point out that amplifiers can be divided into different classes depending on the nature of the loss present. The Langevin source associated with the gain term is fundamental to any amplifier, such as erbium-doped fiber amplifiers (EDFAs) and fiber Raman amplifiers. When amplifiers have loss distributed along the length, a loss noise source must included as well. In the case of an EDFA this loss might arise from linear losses in the fiber as well imperfect inversion of erbium atoms. This last loss mechanism is fundamental to the EDFA operation. In the case of a Raman amplifier, however, there is no such loss term because the Raman intermediate states are virtual levels. The medium is usually transparent even at low-pump levels. In this sense, the Raman amplifier may be thought of as a "perfectly inverted" medium. Similarly, for a silicon Raman amplifier, there is no loss from imperfect inversion. The loss in the amplifying medium consists of the usual linear loss and the nonlinear optical loss described in Section II-A. The nonlinear loss is absent in fiber Raman amplifiers and from this point of view, the analysis of noise in silicon Raman amplifiers is more complicated than that in fiber based devices. The present analysis applies to silicon as well as other semiconductor Raman media.

We can now rewrite the equations for the Stokes wave including the noise sources

$$
\begin{aligned}
\frac{d}{d x} \hat{a}_{S}=\left(\left(\frac{g(x)}{2}\right)-\left(\frac{\gamma^{\prime}(x)}{2}\right)\right) & \hat{a}_{S} \\
& +\sqrt{g(x)} \hat{N}_{G}^{+}+\sqrt{\gamma^{\prime}(x)} \hat{N}_{L} .
\end{aligned}
$$


This equation must be solved numerically, also taking into account the variation of the pump intensity along the waveguide due to the optical losses

$$
\frac{d I_{P}(x)}{d x}=-\gamma I_{P}(x)-\beta I_{P}^{2}(x)-\beta^{\prime} I_{P}^{3}(x) .
$$

\section{Noise Figure of the Amplifier}

The solution of (4) has the form

$$
\begin{aligned}
\hat{a}_{S}(x)= & \exp \left(\left(\frac{1}{2}\right) \int_{0}^{x} d x^{\prime \prime}\left(g\left(x^{\prime \prime}\right)-\gamma^{\prime}\left(x^{\prime \prime}\right)\right)\right) \hat{a}_{S}(0) \\
& +\int_{0}^{x} d x^{\prime} \exp \left(\left(\frac{1}{2}\right) \int_{x^{\prime}}^{x} d x^{\prime \prime}\left(g\left(x^{\prime \prime}\right)-\gamma^{\prime}\left(x^{\prime \prime}\right)\right)\right) \\
& \times\left(\sqrt{g\left(x^{\prime}\right)} \hat{N}_{G}^{+}\left(x^{\prime}\right)+\sqrt{\gamma^{\prime}\left(x^{\prime}\right)} \hat{N}_{L}\left(x^{\prime}\right)\right)
\end{aligned}
$$

We can now calculate the noise figure by evaluating (5) with an input state: $|a\rangle_{S}|0\rangle_{L}|0\rangle_{G}$, where |\rangle$_{S},|\rangle_{L}$, and |\rangle$_{G}$ denote the Stokes, anti-Stokes, and "noise reservoir" states. We find the noise figure of the Raman amplifier by evaluating the mean output photon number and mean photon number fluctuation

$$
F=\frac{T+N_{\text {loss }}+N_{\text {gain }}}{T}+\frac{N_{\text {gain }}\left(T+N_{\text {loss }}\right)}{T^{2}|a|^{2}}
$$

where

$$
\begin{aligned}
T & =\exp \left(\int_{0}^{x} d x^{\prime \prime}\left(g\left(x^{\prime \prime}\right)-\gamma^{\prime}\left(x^{\prime \prime}\right)\right)\right) \\
N_{\text {gain }} & =\int_{0}^{x} d x^{\prime} g\left(x^{\prime}\right) \exp \left(\int_{x^{\prime}}^{x} d x^{\prime \prime}\left(g\left(x^{\prime \prime}\right)-\gamma^{\prime}\left(x^{\prime \prime}\right)\right)\right) \\
N_{\text {loss }} & =\int_{0}^{x} d x^{\prime} \gamma^{\prime}\left(x^{\prime}\right) \exp \left(\int_{x^{\prime}}^{x} d x^{\prime \prime}\left(g\left(x^{\prime \prime}\right)-\gamma^{\prime}\left(x^{\prime \prime}\right)\right)\right)
\end{aligned}
$$

and $|a|^{2}$ is the photon number at the input frequency. For a transform-limited input wave-packet of temporal width $\Delta t$ and frequency width $\Delta \omega$, we can write $|a|^{2}=R / \Delta \omega$, where $R=$ $|a|^{2} / \Delta t$ is the photon rate.

The above expressions are evaluated numerically for various values of the carrier lifetime, which is the critical parameter of the Raman amplifier. It is clear from the above expressions that the nonlinear loss degrades the net gain, but adds to the overall noise.

\section{RESULTS}

We evaluate the above expressions for a waveguide in which a $\mathrm{p}$-n junction is used to sweep out the TPA-generated carriers. We will use the waveguide dimensions and parameters used in [7]: $w=1.5 \mu \mathrm{m}, h=0.75 \mu \mathrm{m}$, and $H=1.55 \mu \mathrm{m}$ (see Fig. 2 inset). For an applied reverse bias voltage of $10 \mathrm{~V}$, the carrier lifetime dependence obtained from 2-D numerical simulations [7] is fitted to the model described by (2), with model parameters $\xi=0.8617, \tau_{H}=1.6 \mathrm{~ns}, n=4.885$, and $I_{\mathrm{TH}}=165 \mathrm{~mW} / \mathrm{cm}^{2}$. The minimum lifetime obtained this way is 22 ps and occurs at moderate pump intensities where carrier removal is not impaired by electric field screening (Fig. 2).

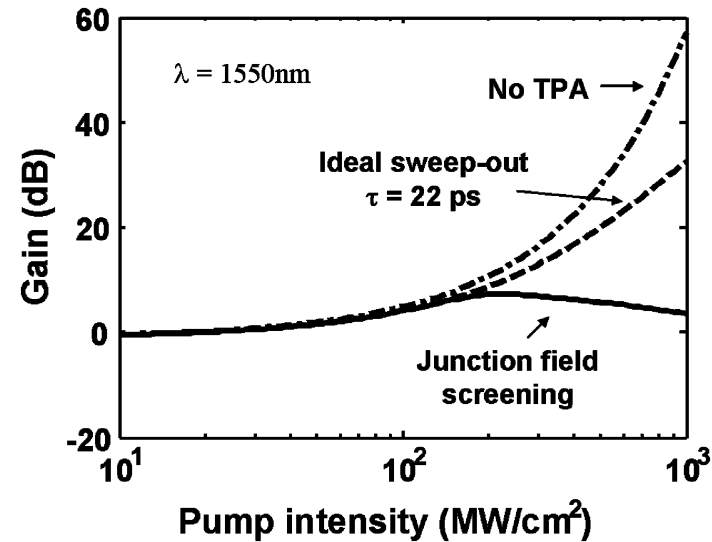

Fig. 3. Amplifier gain versus pump intensity for a device that uses a p-n junction for carrier sweep-out (Fig. 1). Model parameters: length $=1 \mathrm{~cm}$, Raman gain coefficient $=15 \mathrm{~cm} / \mathrm{GW}$, TPA coefficient $=0.7 \mathrm{~cm} / \mathrm{GW}$, free carrier absorption coefficient $=1.45 \times 10^{-17} \mathrm{~cm}^{2}$, linear loss $=1 \mathrm{~dB} / \mathrm{cm}$. TPA: Two photon absorption.

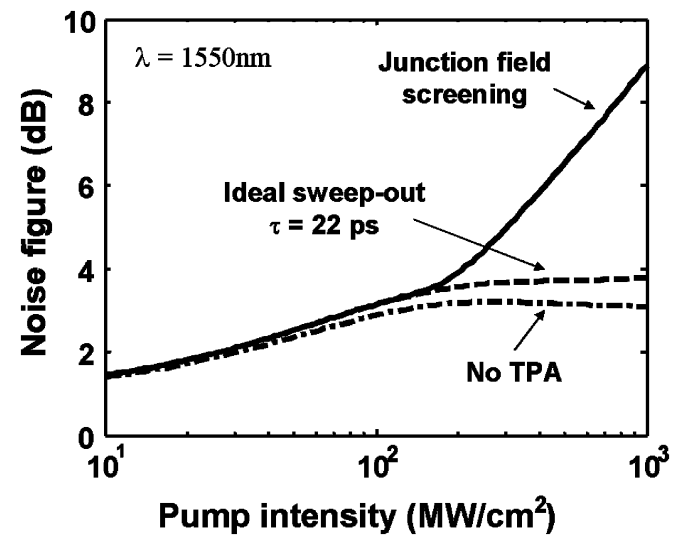

Fig. 4. Amplifier noise figure versus pump intensity for a device that uses a p-n junction for carrier sweep-out (Fig. 1). Model parameters: length $=1 \mathrm{~cm}$, Raman gain coefficient $=15 \mathrm{~cm} / \mathrm{GW}$, TPA coefficient $=0.7 \mathrm{~cm} / \mathrm{GW}$, free carrier absorption coefficient $=1.45 \times 10^{-17} \mathrm{~cm}^{2}$, linear loss $=1 \mathrm{~dB} / \mathrm{cm}$. TPA: Two photon absorption.

Figs. 3 and 4 show the total gain and the noise figure, respectively, for a 1-cm-long waveguide with linear losses of $1 \mathrm{~dB} / \mathrm{cm}$. We used a Raman gain coefficient $g_{R}=15 \mathrm{~cm} / \mathrm{GW}$, a TPA coefficient $\beta=0.7 \mathrm{~cm} / \mathrm{GW}$, and the free carrier absorption cross-section $\sigma=1.45 \times 10^{-17} \mathrm{~cm}^{2}$. For the noise figure calculation, we assumed that the input signal power is large enough so that the second term in (7a) is negligible. This gives the best achievable noise figure which is the objective of this work. The input pump intensity varies from 10 to $1000 \mathrm{~mW} / \mathrm{cm}^{2}$, illustrating the case of ideal carrier removal and removal impaired by electric field screening. For comparison, we also show the case where TPA and free carrier absorption are absent. One can clearly observe the detrimental effect of FCA on the gain and the noise figure. If electric field screening was absent, the device could achieve a noise figure of $\sim 3.5 \mathrm{~dB}$. However, junction field screening results in a marked increase in the noise figure.

Lifetime reduction techniques can also be used to reduce the density of TPA-induced free carriers. Lifetime can be reduced 


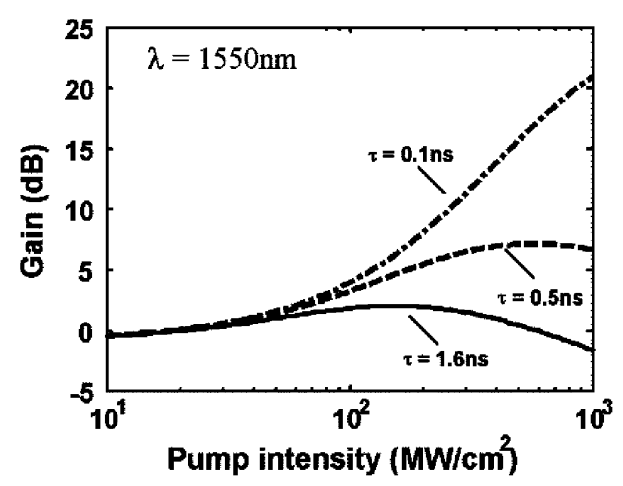

Fig. 5. Amplifier gain versus pump intensity for a device without a p-n junction. The figure illustrates the impact of lifetime reduction if it can be achieved without increasing the loss. Model parameters: length $=1 \mathrm{~cm}$, Raman gain coefficient $=15 \mathrm{~cm} / \mathrm{GW}$, TPA coefficient $=0.7 \mathrm{~cm} / \mathrm{GW}$, free carrier absorption coefficient $=1.45 \times 10^{-17} \mathrm{~cm}^{2}$, linear loss $=1 \mathrm{~dB} / \mathrm{cm} . \tau$ : lifetime.

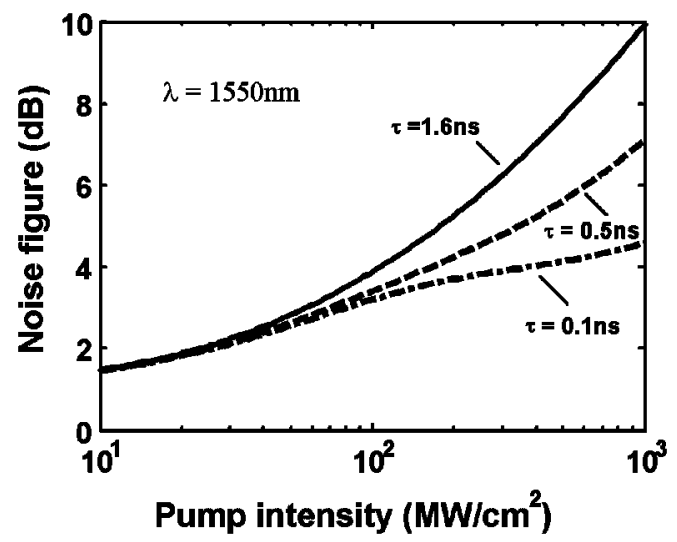

Fig. 6. Amplifier noise figure versus pump intensity for a device without a $\mathrm{p}-\mathrm{n}$ junction. The figure illustrates the impact of lifetime reduction if it can be achieved without increasing the loss. Model parameters: length $=1 \mathrm{~cm}$, Raman gain coefficient $=15 \mathrm{~cm} / \mathrm{GW}$, TPA coefficient $=0.7 \mathrm{~cm} / \mathrm{GW}$, free carrier absorption coefficient $=1.45 \times 10^{-17} \mathrm{~cm}^{2}$, linear loss $=1 \mathrm{~dB} / \mathrm{cm}$. $\tau$ : lifetime.

by scaling down the lateral waveguide dimensions [6], by ionbeam-induced damage, or doping with deep-level impurities. The latter technique is used to create fast silicon step-recovery diodes, to create recombination centers and, hence, to lower the steady-state free carrier density. Ion-beam-induced damage (helium and argon) have recently been applied to silicon waveguides and lifetime reduction has been reported [9], [10]. To show the central role of the intrinsic lifetime of the waveguide, we plot in Figs. 5 and 6 the gain and the noise figure for different values of lifetime. The value of $1.6 \mathrm{~ns}$ is typical for an SOI waveguide with $1 \mu \mathrm{m}$ cross 500 ps has been reported in submicron waveguides [11], [12]. The results indicate that the noise figure is a strong function of carrier lifetime, and show that low-noise amplification requires a lifetime on the order of $100 \mathrm{ps}$. Such a value is reported in [10] in argon-implanted silicon waveguides. The key issue with lifetime reduction techniques is that they may increase the waveguide propagation (linear) losses. The results in Figs. 5 and 6 overestimate the amplifier performance because they assume that the lifetime is reduced without a concomitant increase in waveguide losses-a highly desirable

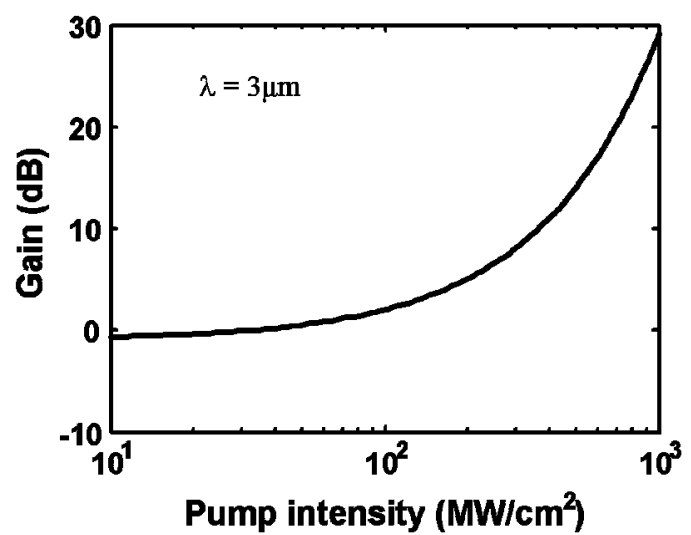

Fig. 7. Amplifier gain versus pump intensity for a silicon Raman amplifier operating at mid-IR wavelength of $3 \mu \mathrm{m}$ where two photon absorption and pump-induced free carrier absorption are absent. Model parameters: length $=$ $1 \mathrm{~cm}$, Raman gain coefficient $=15 \mathrm{~cm} / \mathrm{GW}$, linear loss $=1 \mathrm{~dB} / \mathrm{cm}$.

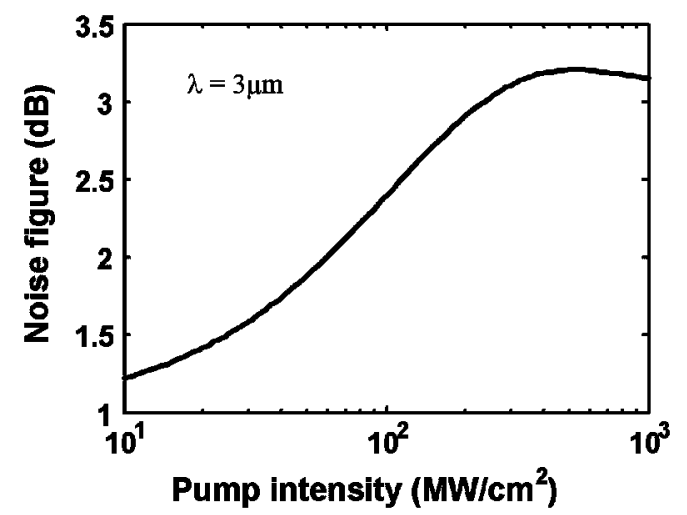

Fig. 8. Amplifier noise figure versus pump intensity for a silicon Raman amplifier operating at mid-IR wavelength of $3 \mu \mathrm{m}$ where two photon absorption and pump-induced free carrier absorption are absent. Model parameters: length $=$ $1 \mathrm{~cm}$, Raman gain coefficient $=7.75 \mathrm{~cm} / \mathrm{GW}$, linear loss $=1 \mathrm{~dB} / \mathrm{cm}$.

outcome that is yet to be experimentally demonstrated. Furthermore, induced crystal damage can also lead to broadening of the Raman linewidth and consequently to reduction of gain. Nevertheless, the model presented here can be used to calculate the noise figure for any combination of lifetime, gain and loss parameters.

We point out that in silicon Raman amplifiers operating at wavelengths greater than the two-photon bandgap $\sim 2.3 \mu \mathrm{m}$, TPA and the ensuing free carrier absorption are absent [13]. For these wavelengths, the energy of two-photons is insufficient to excite an electron from the valence band to the conduction band. Figs. 7 and 8 show the gain and noise figure at the wavelength of $3 \mu \mathrm{m}$. An inverse scaling of Raman gain with wavelength has been assumed, which suggests a gain of $7.75 \mathrm{~cm} / \mathrm{GW}$ at the wavelength of $3 \mu \mathrm{m}$. The impact of TPA-induced free-carrier loss is remarkable: the noise figure does not exceed $3.5 \mathrm{~dB}$ for any pump intensity. These results clearly show the superior performance of silicon Raman devices in the mid-IR spectrum and underscore the case for mid-IR silicon photonics.

The aim of the present analysis has been to determine the limit for the intrinsic noise figure of a silicon Raman amplifier. This 
was achieved by considering the noise contributed by the amplification and dissipative processes that occur in silicon. In other words, the pump laser was assumed to be ideal. In reality, the amount of amplitude noise contributed by the pump laser will depend on the noise characteristics of the particular pump laser and on group velocity dispersion (GVD) in the waveguide. The impact of dispersion on the transfer of pump noise to the Stokes signal has been extensively studied in fiber Raman amplifiers [14]. The pertinent features of pump-noise transfer can be understood by considering a signal that experiences gain from a co-propagating pump. Assuming zero GVD, the same group of signal and pump photons will travel together throughout the waveguide length. Hence, any relative intensity noise (RIN) in pump amplitude will be imparted to the signal amplitude by the Raman gain. However, if the GVD is nonzero, the signal photons will pass through different groups of pump photons as they traverse the gain medium. If the gain medium is long enough or the GVD is large enough, the signal photons will scan several periods of pump amplitude variations, and, hence, the noise will be averaged out. The averaging depends on the GVD and the waveguide length and does occur in fiber Raman amplifiers. It results in suppression of noise fluctuations above a cutoff frequency that is approximately the inverse of the relative group delay between the pump and the signal. Because of the short waveguide length, the noise suppression is not expected to be as significant in silicon Raman amplifiers unless extremely long waveguide lengths are used.

\section{CONCLUSION}

We have calculated the noise figure for a silicon Raman amplifier using a quantum-optical Langevin approach. We find that significant noise degradation is caused by the nonlinear losses in the near infrared wavelengths. The noise figure is a strong function of carrier recombination lifetime and lifetimes on the order of $100 \mathrm{ps}$ or less are desired for a high-fidelity amplifier. In devices that use a p-n junction for carrier sweep-out, the onset of junction field screening is accompanied by a sharp increase in noise figure. The best performance for silicon Raman amplifiers is obtained at the mid-infrared wavelengths greater than $\sim 2.3 \mu \mathrm{m}$, where two photon absorption is absent. In this regime, an intrinsic low-noise figure approaching the theoretical limit of $3 \mathrm{~dB}$ can be expected.

\section{APPENDIX}

The derivation of the noise sources can be found in [8], but we also include it below for completeness. The noise source is derived on the principle that it is needed to conserve the commutator of the optical mode when loss or gain is present in a medium.

Consider a single mode with $\left[\hat{a}_{S}, \hat{a}_{S}^{+}\right]=1$ experiencing gain and add a noise source operator

$$
\frac{d \hat{a}_{S}}{d x}=\left(\frac{1}{2}\right) g \hat{a}_{S}+\hat{F}_{G}
$$

Now demand that

$$
\frac{d\left[\hat{a}_{S}, \hat{a}_{S}^{+}\right]}{d x}=0
$$

which gives

$$
g\left[\hat{a}_{S}, \hat{a}_{S}^{+}\right]+\left[\hat{F}_{G}, \hat{a}_{S}^{+}\right]+\left[\hat{a}_{S}, \hat{F}_{G}^{+}\right]=0 .
$$

The noise operator and the field operator do not commute. To see that, consider the inhomogeneous solution of the propagation equation

$$
\hat{a}_{S}^{I N H}=\int_{-\infty}^{x} d x^{\prime} \exp \left(g\left(x-x^{\prime}\right) / 2\right) \hat{F}_{G}\left(x^{\prime}\right) .
$$

Then we have for the commutator

$$
\begin{array}{r}
{\left[\hat{a}_{S}^{I N H}(x), \hat{F}_{G}^{+}(x)\right]=\int_{-\infty}^{x} d x^{\prime} \exp \left(g\left(x-x^{\prime}\right)\right)} \\
{\left[\hat{F}_{G}\left(x^{\prime}\right), \hat{F}_{G}^{+}(x)\right] .}
\end{array}
$$

Now if the commutator of the noise source is proportional to a delta function with the constant of proportionality equal $\mathrm{C}$, the integration gives

$$
\left[\hat{a}_{S}^{I N H}(x), \hat{F}_{G}^{+}(x)\right]=\left(\frac{1}{2}\right) C .
$$

In order to satisfy (A3), we must have $C=-g$ and the commutator of the noise sources is given by

$$
\left[\hat{F}_{G}\left(x^{\prime}\right), \hat{F}_{G}^{+}(x)\right]=-g \delta\left(x-x^{\prime}\right) .
$$

Notice that the commutator has a negative value. This means that $\hat{F}_{G}(x)$ now acts as a creation operator and $\hat{F}_{G}^{+}(x)$ as annihilation operator. To restore the standard correspondence, we define a new noise source operator such that $\hat{F}_{G}(x)=\sqrt{g} \hat{N}_{G}^{+}(x)$, where

$$
\left[\hat{N}_{G}(x), \hat{N}_{G}^{+}\left(x^{\prime}\right)\right]=\delta\left(x-x^{\prime}\right) .
$$

Now consider the case of loss in the optical mode

$$
\frac{d \hat{a}_{S}}{d x}=-\left(\frac{1}{2}\right) \gamma \hat{a}_{S}+\hat{F}_{L} .
$$

The solution for this situation can be obtained from the results for optical gain by substituting $g \rightarrow-\gamma$ in (A1)-(A7). This yields the commutation relation for the loss noise source

$$
\left[\hat{F}_{L}\left(x^{\prime}\right), \hat{F}_{L}^{+}(x)\right]=\gamma \delta\left(x-x^{\prime}\right) .
$$

In this case, the commutator of the noise source is positive and, therefore, $\hat{F}_{L}(x)$ is a normal creation operator. We use the notation $\hat{F}_{L}(x)=\sqrt{\gamma} \hat{N}_{L}(x)$, where

$$
\left[\hat{N}_{L}(x), \hat{N}_{L}^{+}\left(x^{\prime}\right)\right]=\delta\left(x-x^{\prime}\right) .
$$

\section{ACKNOWLEDGMENT}

This work was supported by the Microsystems Technology Office of DARPA under the EPIC program.

\section{REFERENCES}

[1] O. Boyraz and B. Jalali, "Demonstration of $11 \mathrm{~dB}$ fiber-to-fiber gain in a silicon Raman amplifier," IEICE Electron. Exp., vol. 1, pp. 429-434, 2004. 
[2] T. K. Liang and H. K. Tsang, "Efficient Raman amplification in siliconon-insulator waveguides," Appl. Phys. Lett., vol. 85, pp. 3343-3343, 2004.

[3] X. Qianfan, V. R. Almeida, and M. Lipson, "Time-resolved study of Raman gain in highly confined silicon-on-insulator waveguides," Opt. Exp., vol. 12, pp. 20-20, 2004.

[4] O. Boyraz and B. Jalali, "Demonstration of a silicon Raman laser," Opt. Exp., vol. 12, pp. 5269-5269, 2004.

[5] H. Rong, R. Jones, A. Liu, O. Cohen, D. Hak, A. Fang, and M. Paniccia, "A continuous-wave Raman silicon laser," Nature, vol. 433, pp. 725-725, 2005.

[6] D. Dimitropoulos, R. Jhaveri, R. Claps, J. C. S. Woo, and B. Jalali, "Lifetime of photogenerated carriers in silicon-on-insulator rib waveguides," Appl. Phys. Lett., vol. 86, pp. 071115-071115, 2005.

[7] D. Dimitropoulos, S. Fathpour, and B. Jalali, "Limitations of active carrier removal in silicon Raman lasers and amplifiers," Appl. Phys. Lett., vol. 87, pp. 261108-261108, 2005.

[8] H. A. Haus, Electromagnetic Noise and Quantum Optical Measurements. New York: Springer, 2000.

[9] Y. Liu and H. K. Tsang, "Nonlinear absorption and Raman gain in helium ion implanted silicon waveguides," Opt. Lett., vol. 31, pp. 1714-1716, 2006

[10] T. Tanabe, K. Nishiguchi, A. Shinya, E. Kuramochi, H. Inokawa, M. Notomi, K. Yamada, T. Tsuchizawa, T. Watanabe, H. Fukuda, H. Shinojima, and S. Itabashi, "Fast all-optical switching using ion-implanted silicon photonic crystal nanocavities," Appl. Phys. Lett., vol. 90, pp. 031115-031115, 2007.

[11] R. L. Espinola, J. I. Dadap, R. M. Osgood, Jr, S. J. McNab, and Y. A. Vlasov, "Raman amplification in ultrasmall silicon-on-insulator wire waveguides," Opt. Exp., vol. 12, pp. 3713-3713, 2004.

[12] V. R. Almeida, C. A. Barrios, R. R. Panepucci, M. Lipson, M. A. Foster, D. G. Ouzounov, and A. L. Gaeta, in CLEO CTuFF3, 2004.

[13] B. Jalali, V. Raghunathan, R. Shori, S. Fathpour, D. Dimitropoulos, and O. Stafsudd, "Prospects for silicon mid-IR Raman lasers," IEEE J. Sel. Topics Quant. Electron., vol. 12, no. 12, pp. 1618-1627, Dec. 2006.

[14] C. R. S. Fludger, V. Handerek, and R. J. Mears, "Pump to signal RIN transfer in Raman fiber amplifiers," J. Lightw. Technol., vol. 19, no. 8, pp. 1140-1148, Aug. 2001.

Dimitrios Dimitropoulos received the B.S. degree in electrical engineering from the National Technical University of Athens, Greece, in 2000, and the M.S. and Ph.D. degrees in electrical engineering from the University of California, Los Angeles (UCLA), in 2003 and 2007, respectively.

He is currently a Postdoctoral Research Fellow at UCLA. His current research is in silicon integrated optical devices, particularly silicon Raman lasers, amplifiers, and wavelength converters. His research interests include areas of quantum electronics and photonics, noise in physical systems, information theory, and solid-state physics.

Daniel R. Solli, photograph and biography not available at the time of publication.

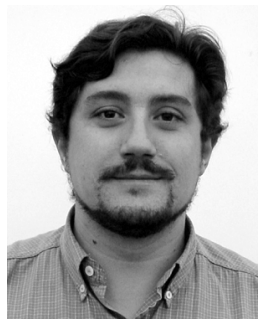

Ricardo Claps was born in Chile, in 1969. He received the B.A. degree in physics from the National University of Mexico (UNAM) in 1993 and the Ph.D. degree in physics from The University of Texas at Austin in 2000.

He was with the Laser Science Institute of Rice University, Houston, TX, as a Postdoctoral Fellow. $\mathrm{He}$ built a trace-gas sensor for ammonia, used in a bioreactor developed for space operations at NASAJohnson Space Center, Houston, TX. While a postdoctoral researcher at the University of California, Los Angeles, he measured stimulated raman scattering in silicon waveguides for the first time (2003). He also discovered the effect of Coherent Anti-Stokes Raman Scattering in silicon. He is now with Neptec Optical Solutions, Fremont, $\mathrm{CA}$, developing novel instrumentation using spectroscopy for the diagnosis and treatment of different diseases, ranging from cardiovascular conditions to diabetes. He is the author of three patents, pending publication.

Ozdal Boyraz (M'96) received the B.S. degree from Hacettepe University, Ankara, Turkey, in 1993, and the M.S. and Ph.D. degrees from the University of Michigan, Ann Arbor, in 1997 and 2001, respectively, all in electrical engineering.

From 2001 to 2003, he was an R\&D Engineer with Xtera Communications, Allen, TX. From 2003 to 2005, he was a Postdoctoral Research Fellow with the Department of Electrical Engineering, University of California, Los Angeles. In August 2005, he joined the Department of Electrical Engineering and Computer Science, University of California, Irvine, as an Assistant Professor. He is the author or coauthor of more than 60 scientific publications and is the holder of three U.S. patents.

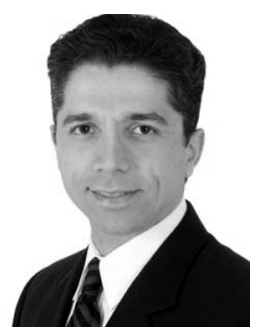

Bahram Jalali is a Professor of electrical engineering and the Director of the Optoelectronic Circuits and System Laboratory at the University of California, Los Angeles (UCLA). From 1988 to 1992, he was a Member of Technical Staff at the Physics Research Division of AT\&T Bell Laboratories, Murray Hill, NJ, where he conducted research on ultrafast electronics and optoelectronics. While on leave from UCLA from 1999-2001, he founded Cognet Microsystems, a Los Angeles-based fiber optic component company. He served as the company's CEO, President, and Chairman from the company's inception through its acquisition by Intel Corporation in April 2001. From 2001-2004, he served as a Consultant to Intel Corporation. He serves on the Board of Trustees of the California Science Center. He has published over 200 scientific papers in and holds six U.S. patents. His current research interests are in silicon photonics and ultrafast photonic signal processing.

Dr. Jalali is a Fellow of Optical Society of America (OSA) and the Chair of the Los Angeles Chapter of the IEEE Lasers and Electro Optics Society (LEOS). In 2005, he was chosen by the Scientific American Magazine as the 50 Leaders Shaping the Future of Technology. He is a member of the California Nano Systems Institute (CNSI). He received the BridgeGate 20 Award for his contribution to the Southern California economy. 